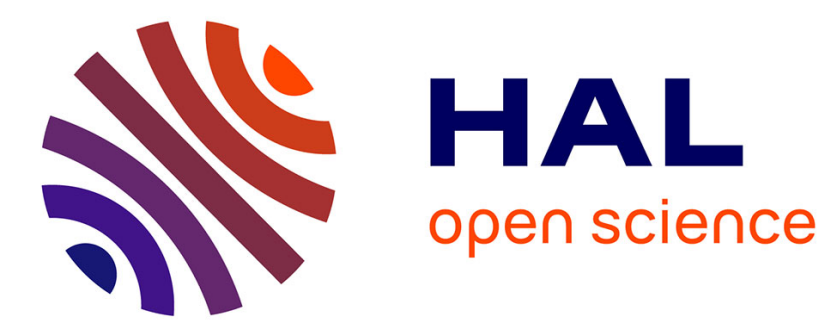

\title{
The Lazy Initialization Multilayered Modeling Framework (NIER track)
}

Fahad Rafique Golra, Fabien Dagnat

\section{To cite this version:}

Fahad Rafique Golra, Fabien Dagnat. The Lazy Initialization Multilayered Modeling Framework (NIER track). ICSE 2011: 33rd International Conference on Software Engineering, May 2011, Honolulu, United States. pp.924 - 927, 10.1145/1985793.1985947 . hal-00623642v2

\section{HAL Id: hal-00623642 \\ https://hal.science/hal-00623642v2}

Submitted on 2 Feb 2017

HAL is a multi-disciplinary open access archive for the deposit and dissemination of scientific research documents, whether they are published or not. The documents may come from teaching and research institutions in France or abroad, or from public or private research centers.
L'archive ouverte pluridisciplinaire HAL, est destinée au dépôt et à la diffusion de documents scientifiques de niveau recherche, publiés ou non, émanant des établissements d'enseignement et de recherche français ou étrangers, des laboratoires publics ou privés. 


\section{The Lazy Initialization Multilayered Modeling Framework (NIER Track)}

\author{
Fahad R. Golra \\ Université Européenne de Bretagne \\ Institut Télécom / Télécom Bretagne \\ fahad.golra@telecom-bretagne.eu
}

\author{
Fabien Dagnat \\ Université Européenne de Bretagne \\ Institut Télécom / Télécom Bretagne \\ fabien.dagnat@telecom-bretagne.eu
}

\begin{abstract}
Lazy Initialization Multilayer Modeling (LIMM) is an object oriented modeling language targeted to the declarative definition of Domain Specific Languages (DSLs) for Model Driven Engineering. It focuses on the precise definition of modeling frameworks spanning over multiple layers. In particular, it follows a two dimensional architecture instead of the linear architecture followed by many other modeling frameworks. The novelty of our approach is to use lazy initialization for the definition of mapping between different modeling abstractions, within and across multiple layers, hence providing the basis for exploiting the potential of metamodeling.
\end{abstract}

\section{Categories and Subject Descriptors}

D.2.2 [Software Engineering]: Design tools and techniques; D.1.5 [Programming Techniques]: Object-Oriented Programming; H.1 [Information Systems]: Models and principles

\section{General Terms \\ Design, Standardization}

\section{Keywords}

Metamodeling, Strict metamodeling, Instantiation, LIMM

\section{INTRODUCTION}

Modeling was once considered a tool to understand the System Under Study (SUS) but recently it has emerged out as a key activity of system development. This led to the rapid and incremental development of UML. However UML restricts modeling to 1) a 4 layers hierarchy, 2) 'strict' metamodeling (a model element must be an instance of exactly one meta-model element), and 3) the exclusive use of instance-of relationship amongst adjacent modeling layers. Holding to backward compatibility, UML2.0 could not unleash the complete potential of metamodeling. Different researchers have been using its evolution mechanism (UML Profiles) to add up to its capabilities [6]. Some recent approaches of metamodeling (deep instantiation, power type based modeling, etc.) question its shortcomings and present their own methodologies $[2,1]$. However none of these approaches succeeded in replacing or influencing UML.

In order to exploit the potential of metamodeling both at micro level (inside a model) and macro level (between models), LIMM is presented as an object oriented multilayered modeling language that aims to provide a better support for the definition of different DSLs. The usage of lazy initialization in modeling is a novel idea that adds a lot of flexibility for the modelers, without introducing any functional limitations. In our proposal, the designer of a model can specify for each model element if it is at meta level, at application level or it is a data. To achieve this goal, flags are associated to model elements to control the way they can be used in the subsequent layers. A flag can take three different values to restrict, allow or force the initialization of a model element in the next adjacent layer.

This paper is structured as follows. Section 2 explains the multilayered modeling framework and describes the recent associated endeavors. Section 3 describes the LIMM framework. Section 4 presents the metamodel for LIMM framework. Finally Section 5 outlines the conclusions and future work.

\section{MULTILAYERED MODELING}

When modeling started to mature, modeling languages themselves needed to be modeled (language-ware). This led to a shift from traditional bi-layered modeling towards multilayered modeling hierarchy. UML modeling framework as defined in MOF rests on a 4 layers hierarchy. Though UML holds the industry, still some modelers (specially the metaCASE tool designers) face some problems using it. The well known problems of this framework are Ambiguous Classification, Replication of Concepts, Structure redundancies and problems with metalevels $[4,1,2,7]$.

One of most discussed problems in literature regarding the UML modeling framework is its incompetence to differentiate between the logical and the physical flavors of the instance-of relationship. As illustrated in Figure 1(a), the document AR2010 is an instance-of class Document, which is an instance of both ResourceType and Class, where the two instantiations are not the same. The first instantiation relationship is a logical relationship mapping an instance to a concept of the system under study (SUS), whereas the second relationship indicates its physical existence as a class. Thus the concept of object/type facets is addressed in MOF, but is not very well defined. The term clabject coined by Atkinson refers to this concept [1].

A unique notion of instance-of relationship between two layers seems unable to portray the concept of class as an instance. Power type based modeling proposed to use instantiation to carry on the instance facet to the subsequent modeling layer, and inheritance to carry the class facet [2]. In this framework, a class (such as Class in Figure 1(b)) has 


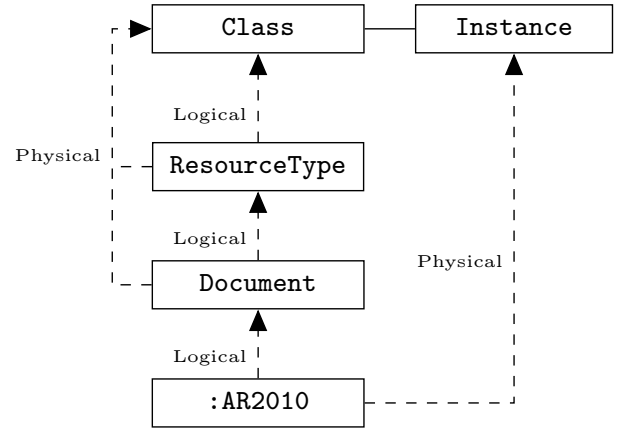

(a) UML

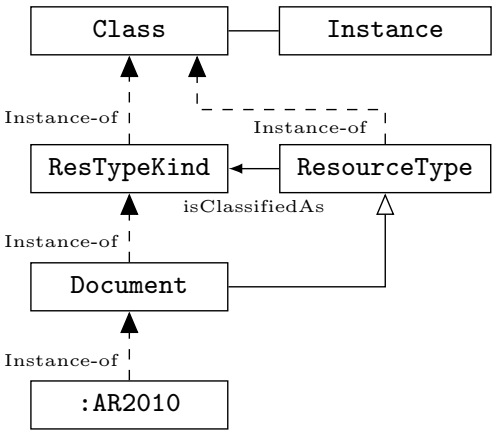

(b) Power Types

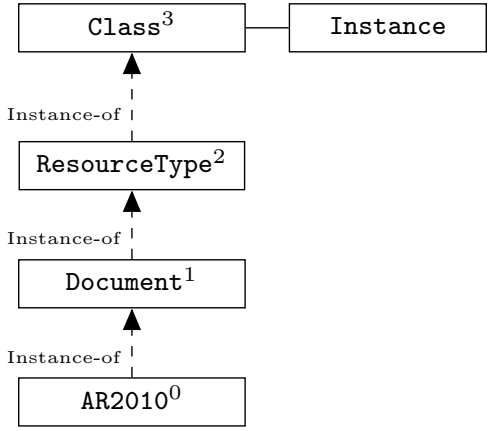

(c) Deep Instantiation

Figure 1: Comparative Example in different Multilayered modeling frameworks

two instances in the subsequent modeling layer. First, the power type, ResTypeKind, carries the object facet to the next level using the instance-of relationship. Second, the partitioned type, ResourceType, assumes the class facet and is intended to be the super class of the power type's instance (Document in the example). Instead of having a simple typeinstance relationship like in traditional modeling, it creates a triplet of the power type, the partitioned type and the class at a lower modeling layer, as depicted in Figure 1(b).

The drawback of power type based modeling is its inability to define associations in terms of clabjects. All the complexity of partitioning the power type from partitioned type in order to classify the different facets is transferred to the semantics of the relationship isClassifiedAs, which lacks concrete explanation. Furthermore, the inheritance relationship across different layers in the architecture makes it quite complex to draw the boundaries of a modeling layer.

In contrast to power type based modeling, deep instantiation based modeling consents to defer the instantiation for a "known" number of layers $[1,4]$. Deep instantiation uses the concept of potency, where each association, attribute and class is assigned a potency. The potency amounts to the number of levels an artifact can deny instantiation. Thus if an attribute is assigned a potency of 2 , and it resides at layer $M_{n}$, then it would not be instantiated till $M_{n-2}$. As it gets down to $M_{n-2}$, it will get instantiated and become a slot. Deep instantiation addresses the dual nature (class and instance facets) of the classes using this potency. An example of deep instantiation can be viewed in Figure 1(c).

One of the major drawbacks of deep instantiation is that one has to know in advance, the number of layers each artifact should resist instantiation. The metamodeler must anticipate the uses of its metamodel and fix the potency of its elements. This fixed potency eventually takes the flexibility out of the reach of the application modeler, who is constrained to follow it.

Álvarez et al. suggested that instead of a linear modeling hierarchy, one should follow the nested hierarchy [5]. In such a hierarchy, the level $M_{0}$ is adjacent to both $M_{1}$ and $M_{2}$ layers. So the instance at level $M_{0}$ can have instance-of relationships both from $M_{1}$ and $M_{2}$. The essence of nested layers is that it is recursive in nature and has a conforming mapping structure called the G-mapping. The drawback of using such a multi layer structure is that, though it works fine for 3 layers, but as it progresses to more layers, the original benefit being sought is lost. Thus it is restricted to a maximum of 4 layers. And the problem of ambiguous classification still remains; as the only relationship allowed is instance-of and no well defined differentiation among the different flavors of instance-of relationship is discussed.

A detailed study of the existing multilayered modeling frameworks shows that each of these techniques tries to resolve a part of the explored shortcomings of UML. None of these techniques, other than UML, gained popularity in the software industry because of the respective drawbacks detailed above. For these reasons, we propose a general multilayered modeling framework influenced by the previously presented approaches but relying on a lazy initialization mechanism inspired from functional programming.

\section{LAZY INITIALIZATION}

The Lazy Initialization Multilayer Modeling (LIMM) takes its inspiration from lazy functional programming, where evaluation may be delayed until required. Using lazy initialization helps clarify the instance-of relationships between the layers and their classification.

In UML, the links in the data models are the instances of the associations in the model at upper layer; same goes for the slots which are the instances of the attributes in the model at upper layer. But the instance-of relationship which bypasses the layers is not well explained. LIMM uses a special sort of abstraction known as interconnection to specify the different relationships that bypass the model boundaries. This framework gives a proper justification and sound basis to various relationships within a model or amongst different models, residing either on same or different abstraction levels.

We argue against deep instantiation because one cannot decide before hand, the number of layers after which a model element is to be initialized. Indeed, we believe that the number of layers cannot be fixed and must result from the choice of the designer. Furthermore, each layer is usually built by a different designer, making it impossible to predict when a model element will reach its object layer (where it is initialized). Our proposal is that at a certain layer, the designer must specify the initialization scheme of model elements in the subsequent layer. The initialization scheme can either forbid the initialization or devolve the decision, to choose the number of layers before initialization, to the designer of the subsequent layer.

The flag value of the associated model element specifies whether it is going to be initialized in the subsequent layer or not. This flag can be null or have three different values: 2 for Restrict, 1 for Relax or 0 for Force. A null flag describes an 
instantiated model element. While developing a model, the flags of the meta-elements in the reference model are tested to determine which elements must or may be initialized at current layer. A flag value 2 (Restrict) specifies a model element that cannot be initialized in the subsequent layer (where the flag value can shift to 2,1 or 0 ). A flag value 1 (Relax) allows the initialization of a model element in the subsequent layer (where its flag value can become 1,0 or null). Lastly, a flag value 0 (Force) forces the designer of the subsequent layer to initialize the model element (moving its flag to null).

Models being model elements maintain their own flags to specify their abstraction level. Models having a flag value 2 are at some metalevels whereas the models having the flag value 0 are the user models. Models having a flag value 1 can be intermediate level models that can/cannot be initialized. A model contains classes and these classes contain attributes. This containment hierarchy constrains flags: the flag value of a container must be greater than or equal to the flag value of its contained elements. Data models or object models do not carry a flag, so they have a null value. The flag values of a model can either be specified by the designer or calculated automatically.

LIMM uses a two dimensional modeling framework with a horizontal division between the core multilayering metamodel and the linear modeling hierarchy, which is further divided into multiple modeling layers. The core multilayering metamodel is the LIMM metamodel that defines the language in which the models placed in the various layers are defined. This is a top level meta-metamodel that defines itself. Our numbering of the linear modeling hierarchy, in contrast to UML, keeps augmenting from the topmost layer, $M_{0}$, to the subsequent layers up to $M_{n}$.

\section{LIMM METAMODEL}

LIMM metamodel presents two common models; a user model and a reference model, which are connected through interconnections, as shown in Figure 2. A reference model can be referenced by another reference model or by a data model. A data model is a model which is not referenced by any other model. The core multilayering metamodel does not have a flag because all other models in its linear hierarchy are instance-of this model, thus all the physical instanceof relationships are implicit in this model. The dual nature (type/instance) of a model element is described in terms of initialized and non-initialized contained model elements. The common model is itself a model element to support abstraction hierarchies. All the meta-metamodels for defining new DSLs are presented as an extension of the core multilayering metamodel. This allows them to utilize multiple layers for model definitions.

Channels and Items are both model elements, which are used to relate a model to the graph semantics. They are kept open to extension for new abstractions in order to support the definition of new DSLs. One of the hallmarks of UML was the visual representation through adding semantics to the graphs. LIMM uses the same methodology but maintains a distinction between the channel and interconnection. A channel is a relationship between two model elements within a model, whereas an interconnection is a relationship between elements of different models. Thus the semantics of relationships crossing the boundaries of a model is different from those who do not. The classification of $i n$ -

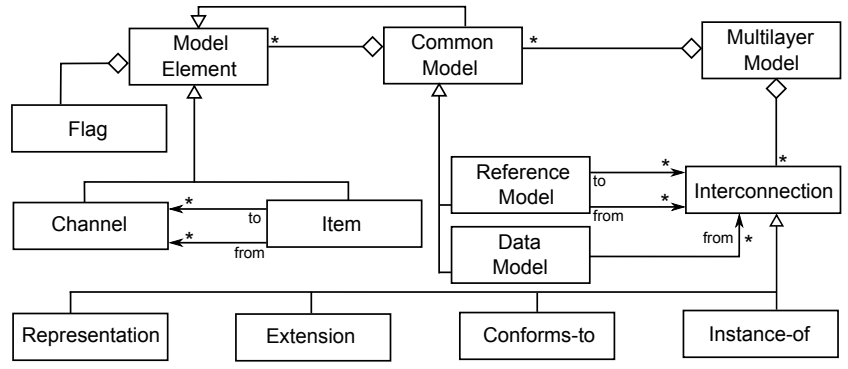

Figure 2: Minimal LIMM metamodel

terconnection is done in terms of abstraction levels. A Cross Layer Interconnection (CLI) relates two model elements residing on different layers, whereas a Same Layer Interconnection (SLI) relates the model elements residing on the same layer. Instance-of and Conformity are CLIs that contravene the strict metamodeling of UML, where the only relationship across layers can be instance-of.

A DSL for metamodel specification, Kernel MetaMetaModel (KM3) [3], defines the structure of a model using directed multigraphs. We follow their definition and extend it further for describing our specification. Let $G_{M}=$ $\left(N_{M}, E_{M}, \Gamma_{M}\right)$ be the directed multigraph for the common model $M$, where

- $N_{M}$ is the set of nodes (representing the Items);

- $E_{M}$ is the set of edges (representing the Channels);

- $\Gamma_{M}$ is the function from $E_{M}$ to $N_{M} \times N_{M}$ associating an edge to its extremities.

A common model $M$ is represented as $M=\left(G_{M}, R, \kappa_{M}\right)$, where

- $R$ is the reference model of $M$. The associated graph of this reference model is $G_{R}=\left(N_{R}, E_{R}, \Gamma_{R}\right)$.

- $\kappa_{M}$ is a function from $N_{M} \cup E_{M}$ to $N_{R} \cup E_{R}$. It relates the elements of the model $M$ to the elements of the reference model $R$ (meta-elements), thus representing the explicit logical relationships.

Having the common model and reference model in hand, we are able to represent the multilayer metamodel $M L=$ $\left(G_{M L}, C M M, \kappa_{M L}\right)$, such that

- $G_{M L}=\left(N_{M L}, E_{M L}, \Gamma_{M L}\right)$ is a directed multigraph for the multilayered model, where

- $N_{M L}$ is the set of nodes of the graph representing the elements of multilayer model, $N_{M} \cup N_{R}$.

- $E_{M L}$ is the set of edges of the graph representing the associations in multilayer model, $E_{M} \cup E_{R}$.

- $\Gamma_{M L}$ is the function from $E_{M L}$ to $N_{M} \cup E_{M} \times N_{R} \cup$ $E_{R}$ associating this edge to its extremities.

- $C M M$ is the core multilayering metamodel which defines itself. It is the topmost level of hierarchy, where the meta-metamodel is defined recursively by itself.

- $\kappa_{M L}$ is a function from $N_{M L} \cup E_{M L}$ to $N_{C M M}$, relating all the model elements and relationships of the multilayer model to the nodes of core multilayering metamodel representing the physical relationships. These relationships are implicit in the visual representation. 


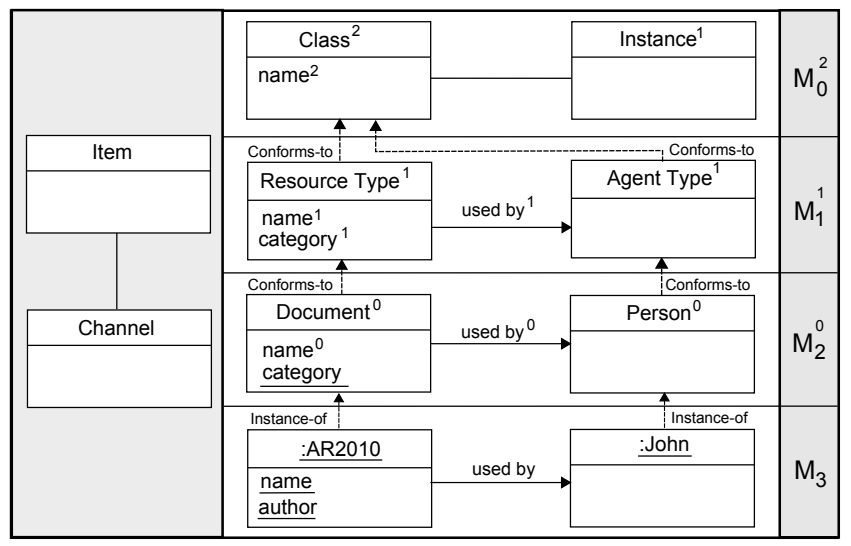

Figure 3: Example in LIMM

A reference model is referenced by a data model from a lower layer but at the same time it may refer to some other reference model (acting itself as data model). The elements of the reference model $R$ are hence meta-elements for the elements of the models residing in the subsequent modeling layer. The CLIs (instantiation and conformity) can be defined when $M$ and $R$ are both at different layers, whereas SLIs can be defined when $M$ and $R$ are both at the same layers. For a model $M$, a partial function $f_{0}$ gives the flag value of its model elements if it is not null. Thus, $\forall e \in N_{M} \cup E_{M}, f_{M}(e) \in\{0,1,2\}$.

Conforms-to relationship $(\mu)$ is defined for LIMM as a Cross Level Interconnection (CLI) when,

$$
\mu=\left\{i \in \kappa_{M} \mid \operatorname{dom}(i) \subseteq \operatorname{dom}\left(f_{M}\right)\right\}
$$

Instance-of relationship $(\eta)$ is defined for LIMM as a Cross Level Interconnection (CLI) when,

$$
\eta=\left\{i \in \kappa_{M} \mid \operatorname{dom}(i) \cap \operatorname{dom}\left(f o_{M}\right)=\emptyset\right\}
$$

The example used to compare other multilayered modeling frameworks is continued in Figure 3 where a part of object oriented metamodel lies at $M_{0}$ layer. The ResourceType class at $M_{1}$ layer conforms to the class Class at $M_{0}$. The flag value of the attribute name is not initialized till $M_{3}$, whereas category is initialized at $M_{2}$. In this example, AR2010 has an instance-of relationship to Document, which in turn has a conforms-to relationship with ResourceType. The usedby link at $M_{3}$ is initialized from the usedby association at $M_{2}$. The flag values on the models and model elements show that they belong to meta layer, application layer or data layer. The instance facet of the class Document can be viewed from category (slot), whereas the class facet from name (attribute). All the relationships explicitly defined are the logical relationships, whereas the physical relationships are implicit in the visual representation, from each model to the core multilayering metamodel, adjacent to all the the layers of the multilayer model.

\section{CONCLUSION}

Different multilayered modeling frameworks have been explored to stress their deficiencies and strengths. We proposed to capture the semantics of a model using graphs, where the semantics of the instance-of and conforms-to relationships can be clarified. A metamodel for the lazy initialization multilayered modeling framework is presented, where lazy initialization schemes are used to resolve the issues faced by multilayered models. LIMM framework supports the definition of new DSLs that need the usage of multiple abstraction levels. In addition, this novel framework improves the level of expression in multilayered modeling by adding semantics to the mappings across layers, which would in turn facilitate model validations. A vital impact on the designer's approach would be the enhanced control over his model to guide the development, without forcing any unnecessary restrictions to the subsequent layer designers. Having models as model elements helps add semantics to the mappings amongst the models also. This inter-model mapping would have a considerable impact in assisting model management.

The focus of MDE is to exploit domain modeling by improving compatibility and mappings amongst models. We are looking forward to add semantics to the mapping functions between the model elements. This would further assist the automation of software development processes for multimetamodel applications. For the future work, this multilayered modeling framework would also help to build transformation hierarchies. Having both the model hierarchies and transformation hierarchies would allow LIMM framework to exploit the potential of MDE.

\section{REFERENCES}

[1] C. Atkinson and T. Kuhne. Rearchitecting the UML Infrastructure. ACM Transactions on Modeling and Computer Simulation, 12(4):290-321, 2002.

[2] C. Gonzalez-Perez and B. Henderson-Sellers. A Powertype-based Metamodelling framework. Software and Systems Modeling, 5:72-90, 2006.

[3] F. Jouault and J. Bézivin. KM3: A DSL for Metamodel Specification. In Formal Methods for Open Object-Based Distributed Systems, volume 4037 of $L N C S$, pages 171-185. Springer Berlin / Heidelberg, May 2006.

[4] T. Kuhne. Understanding Metamodeling. In Proc. of the 27th International Conference on Software Engineering (ICSE), pages 716 - 717, May 2005.

[5] J.Álvarez, A. Evans, and P. Sammut. Mapping between levels in the metamodel architecture. In Proc. UML The Unified Modeling Language. Modeling Languages, Concepts and Tools, pages 34-46. Springer, 2001.

[6] J. Rumbaugh, I. Jacobson, and G. Booch. The Unified Modeling Language Reference Manual. Addison-Wesley, 1999.

[7] D. Varró and A. Pataricza. VPM: A visual, precise and multilevel metamodeling framework for describing mathematical domains and UML (the mathematics of metamodeling is metamodeling mathematics). Software and Systems Modeling, 2(3):187-210, October 2003. 